\title{
Laboratory Tests in the Detection of Extended Spectrum Beta-lactamase Production: National Committee for Clinical Laboratory Standards (NCCLS) Screening Test, the E-Test, the Double Disk Confirmatory Test, and Cefoxitin Susceptibility Testing
}

Pedro A. d'Azevedo ${ }^{1}$, Ana Lúcia S. Gonçalves ${ }^{2}$, Maiara I. Musskopf ${ }^{1}$, Carina G. Ramos ${ }^{1}$ and Cícero A. G. Dias ${ }^{1}$
Federal Faculty of Medical Sciences Foundation of Porto Alegre ${ }^{1}$; Central Laboratory of Clinical Analysis of Irmandade Santa Casa de Misericórdia of Porto Alegre ${ }^{2}$; Porto Alegrel RS, Brazil

\begin{abstract}
Extended spectrum beta-lactamase (ESBL) production by Klebsiella sp. and $E$. coli is an emerging problem. In this study, 107 clinical isolates ( $53 \mathrm{E}$. coli, $47 \mathrm{~K}$. pneumoniae and $7 \mathrm{~K}$. oxytoca) screened as ESBL producers by the NCCLS disk diffusion procedure were submitted to a double disk confirmatory test (DDT) and to the E-test double strip for confirmation of ESBL production by demonstration of clavulanic acid inhibition effect (CAIE). Only 72/107 (67\%) of the isolates were confirmed as ESBL producers by DDT, with diverse results among species. By the E-test, 58/ $107(54 \%)$ isolates were confirmed as ESBL producers, and 18/107 (17\%) were not determinable. Susceptibility to cefoxitin was found in $57 / 68$ (83\%) of strains that did not show CAIE. ESBL detection remains a controversial issue and clinical laboratories are in need of a simple and effective way to recognize strains with this kind of resistance.

Key Words: ESBL, double disk confirmatory test, cefoxitin susceptibility testing, screening test.
\end{abstract}

The extended spectrum beta-lactamases (ESBLs) enable certain Gram-negative bacteria to inactivate cephalosporins as well as broad-spectrum penicillins and monobactams (aztreonam). The microorganisms that produce ESBLs most frequently are Klebsiella sp. and E. coli. ESBL production by Gram-negative organisms is an emergent, worldwide problem [1-6] and the presence of these enzymes has an impact on the efficacy of $\beta$-lactam therapy $[7,8]$.

ESBLs can present variations in the in vitro pattern of resistance to $\beta$-lactam agents conferred to the Received on 13 May 2004; revised 17 August 2004.

Address for correspondence: Dr. Pedro A. d'Azevedo, Fundação Faculdade Federal de Ciências Médicas de Porto Alegre, Rua Sarmento Leite 245/211, Porto Alegre, RS 90050170, Brazil E-mail: pedroaze@fffcmpa.tche.br

This study was presented in part at the $102^{\text {nd }}$ General Meeting of American Society for Microbiology, Salt Lake City, Utah, 2002 (Abstract C105).

The Brazilian Journal of Infectious Diseases 2004;8(5):372-377 (C) 2004 by The Brazilian Journal of Infectious Diseases and Contexto Publishing. All rights reserved. samples that encode them [9]. Some enzymes (TEM3 and SHV-2) confer high levels of resistance to cephalosporins, whereas others, such as TEM-7 and TEM-12, confer low levels of resistance, which possibly makes it even more difficult to detect them through the susceptibility tests routinely used in microbiology laboratories. Clinical laboratories must accurately recognize ESBL producers to better support therapy. In 1999, the National Committee for Clinical Laboratory Standards issued recommendations for ESBL detection in E. coli and Klebisella sp. [10]. Recommendations include a screening test, followed by a confirmatory test, both adapted to diffusion and dilution formats. The confirmatory test is based on the demonstration of inhibition by clavulanic acid. However, other mechanisms of $\beta$-lactam resistance, including AmpC type enzymes [11,12], porin changes [13-15], and variants of the original ESBL enzymes [16,17], may be present and even coexist with ESBL, interfering in the results of these tests. 
The aim of this study was to evaluate phenotypic tests, the E-test for detection of ESBL, the Double Disk and cefoxitin susceptibility testing for ESBL production in E. coli and Klebsiella sp.

\section{Material and Methods}

\section{Bacterial strains}

A total of 107 clinical isolates (53 Escherichia coli, 47 Klebsiella pneumoniae and 7 Klebsiella oxytoca) were included in the study. Isolates were obtained from patients at Complexo Irmandade Santa Casa de Misericórdia, a 1,100 bed, tertiary care, university hospital, from June 99 to December 99, and identified by the Negative Combo 21 Panel (DadeBehring, West Sacramento, Califórnia, USA). All isolates were screened as ESBL producers by the NCCLS disk diffusion procedure [10]. Isolates showed reduced zones of inhibition to at least one of the following antimicrobials: ceftazidime $30 \mu \mathrm{g}(\mathrm{CAZ}) \leq$ $22 \mathrm{~mm}$, ceftriaxone $30 \mathrm{mg}(\mathrm{CRO}) \leq 25 \mathrm{~mm}$, aztreonam $30 \mu \mathrm{g}$ (ATM) $\leq 27 \mathrm{~mm}$ and cefotaxime $30 \mu \mathrm{g}(\mathrm{CTX}) \leq 27 \mathrm{~mm}$.

Quality control was performed using E. coli ATCC 25922, and Klebsiella pneumoniae ATCC 700603.

\section{Susceptibility testing}

Confirmation of ESBL phenotype. Double Disk Confirmatory Test (DDCT): The recommendations of Jarlier et al., (1988) were followed with modifications, and of NCCLS (2001) using ceftazidime + clavulanic acid $(\mathrm{CAZ}+\mathrm{CA})$; cefotaxime + clavulanic acid $(\mathrm{CTX}+\mathrm{CA})$.

E-test

The determination of the Minimum Inhibitory Concentration (MIC) was performed through the technique of the E-test (AB Biodisk, Solna, Sweden), on double strips containing ceftazidime $(0.5-32 \mu \mathrm{g} / \mathrm{mL})$ and ceftazidime/ clavulanic acid $(0.064-4 \mu \mathrm{g} / \mathrm{mL})$ in
Mueller-Hinton agar. Isolates were considered ESBL producers when clavulanate caused $\mathrm{a} \geq 3$ twofoldconcentration decrease (ratio $\geq 8$ ) in the MIC. Additionally, a strain was considered an ESBL producer if a phantom zone or a deformation of the ceftazidime zone could be observed, independent of the ratios or MICs. The outcome of the test was indeterminate when both MICs were outside the test range of the test device. This phenomenon suggests the presence of an inhibitor-resistant TEM or AMPC enzymes. Manufacturer's instructions were followed for performing and interpreting the test.

\section{Susceptibility to cefoxitin}

Sixty-eight samples of E. coli and Klebsiella sp. were used, which were positive in the ESBL screening test yet were negative in the confirmatory test. The samples were submitted to a cefoxitin susceptibility test $(30 \mu \mathrm{g})$, according to Steward et al. [21].

\section{Results and Discussion}

Table 1 shows the results of the DDCT using ceftazidime, cefotaxime, and both drugs. Overall, the clavulanic acid inhibition effect (CAIE) was observed in $72 / 107(67 \%)$ of the isolates originally screened as ESBL producers by disk diffusion. CAIE was more frequently observed among isolates of $K$. pneumoniae (75\%), however when $E$ coli and $K$. oxytoca were tested $62 \%$ and $57 \%$ of the isolates, respectively, were confirmed as ESBL producers. Demonstration of CAIE was more evident with CTX-CA. A strategy based exclusively on CAIE by CAZ-CA would have confirmed only 17/53 (32\%) of E. coli, 24/47 (51\%) of $K$. pneumoniae, and $1 / 7$ (14\%) of $K$. oxytoca isolates. Results show that confirmation of ESBL production by DDCT varies according to the substrate used (CTX or CAZ), and that the screening procedure presents a variable performance when compared to the DDCT, according to the organism tested. Better results were obtained when $K$. pneumoniae was tested. 
Table 1. Results of the Double Disk Confirmatory Test with ceftazidime (CAZ) + clavulanic acid (CA) and cefotaxime (CTX) + clavulanic acid (CA) in isolates of E. coli, Klebsiella pneumoniae, and Klebsiella oxytoca screened as extended spectrum beta-lactamase producers by disk diffusion

\begin{tabular}{|c|c|c|c|c|c|c|c|c|c|}
\hline \multirow[t]{2}{*}{ Organism } & \multicolumn{3}{|c|}{$\mathrm{CAZ}+\mathrm{CA}$} & \multicolumn{3}{|c|}{$\mathrm{CTX}+\mathrm{CA}$} & \multicolumn{3}{|c|}{$\mathrm{CAZ}+\mathrm{CA}$ or $\mathrm{CTZ}+\mathrm{CA}$} \\
\hline & $\mathbf{P}(\%)$ & $\mathbf{N}(\%)$ & $\mathrm{T}(\%)$ & $\mathbf{P}(\%)$ & $\mathbf{N}(\%)$ & $\mathrm{T}(\%)$ & $\mathbf{P}(\%)$ & $\mathbf{N}(\%)$ & $\mathbf{T}(\%)$ \\
\hline E. coli & $17(32.1)$ & $36(67.9)$ & $53(100)$ & $25(47.2)$ & $28(52.8)$ & $53(100)$ & $33(62.3)$ & $20(37.7)$ & $53(100)$ \\
\hline K.pneumoniae & $24(51.1)$ & $23(48.9)$ & $47(100)$ & $29(61.7)$ & $18(38.3)$ & $47(100)$ & $35(74.5)$ & $12(25.5)$ & $47(100)$ \\
\hline Koxytoca & $1(14.3)$ & $6(85.7)$ & $7(100)$ & $4(57.1)$ & $3(42.9)$ & $7(100)$ & $4(57.1)$ & $3(42.9)$ & $7(100)$ \\
\hline Total & $42(39.3)$ & $65(60.7)$ & $107(100)$ & $58(54.2)$ & $49(45.8)$ & $107(100)$ & $72(67.3)$ & $35(32.7)$ & $107(100)$ \\
\hline
\end{tabular}

Table 2. Results of E test strips containg ceftazidime (CAZ) and ceftazidime + clavulanic acid (CAZ+CA) in isolates of E. coli, Klebsiella pneumoniae, and Klebsiella oxytoca screened as extended spectrum beta-lactamase (ESBL) producers by disk diffusion

\begin{tabular}{lcrrrr}
\hline & & \multicolumn{3}{c}{ E-test strips containing CAZ and CAZ+CA } & \\
& Pos $(\boldsymbol{\%})$ & Neg $(\%)$ & ND $^{\mathbf{a}}$ & Induction $^{\text {Total }}$ & Totar \\
\hline E. coli & $25(46.9)^{\mathrm{b}}$ & $15(28.3)$ & $12(22.6)$ & $1(1.89)$ & $53(100)$ \\
K. pneumoniae & $31(65.9)$ & $12(25.5)$ & $3(6.38)$ & $1(2.13)$ & $47(100)$ \\
K. oxytoxa $_{\text {Total }}$ & $2(28.6)^{\mathrm{b}}$ & $2(28.6)$ & $3(42.9)$ & 0 & $7(100)$ \\
& $\mathbf{5 8}(\mathbf{5 4 . 2 )}$ & $\mathbf{2 9}(\mathbf{2 7 . 1 )}$ & $\mathbf{1 8}(\mathbf{1 6 . 8})$ & $\mathbf{2 ( 1 . 8 7 )}$ & $\mathbf{1 0 7}(\mathbf{1 0 0 )}$ \\
\hline
\end{tabular}

${ }^{a}$ Not determinable, due to off scale results (below the test ranges).

b One isolate showing "phantom" inhibition zone, compatible with ESBL production.

Table 3. Results of cefoxitin susceptibility tests applied on isolates that did not show clavulanic acid inhibitory effect by double disk confirmatory test

\section{Cefoxitin}

Susceptible Intermediate Resistant Total

\begin{tabular}{lrrrr}
\hline Escherichia coli & 18 & 1 & 1 & 20 \\
Klebsiella pneumoniae & 10 & 0 & 2 & 12 \\
Klebsiella oxytoca & 3 & 0 & 0 & 3 \\
Total & $\mathbf{3 1}$ & $\mathbf{1}$ & $\mathbf{3}$ & $\mathbf{3 5}$ \\
\hline
\end{tabular}


Table 4. Results of cefoxitin susceptibility tests applied on isolates that did not show clavulanic acid inhibitory effect by E test strip containing ceftazidime (CAZ) and ceftazidime + clavulanic acid (CAZ+CA)

\begin{tabular}{llcccr}
\hline & E-test results & Susceptible & $\begin{array}{c}\text { Cefoxitin } \\
\text { Intermediate }\end{array}$ & Resistant & Total \\
\hline E. coli & Negative & 13 & 1 & 1 & 15 \\
& ND $^{\mathrm{a}}$ & 11 & 0 & 1 & 12 \\
Klebsiella pneumoniae & Induction & 1 & 0 & 0 & 1 \\
& Negative & 10 & 1 & 1 & 12 \\
& ND & 3 & 0 & 0 & 3 \\
Klebsiella oxytoca & Induction & 0 & 0 & 1 & 1 \\
& Negative & 2 & 0 & 0 & 2 \\
& ND & 3 & 0 & 0 & 3 \\
& Induction & 0 & 0 & 0 & 0 \\
\hline
\end{tabular}

${ }^{a} \mathrm{ND}=$ not determinable, due to off scale results (below the test ranges).

The quantitative format, using E-test strips containing CAZ and CAZ+CA, had problems in confirming ESBL production (Table 2 ). The CAIE could be demonstrated in only 58/107 (54\%) of the isolates and 18/107 (17\%) were not determinable due to off-scale results (all results below the test ranges). This was a limitation of the quantitative format that was present in all species studied, since $23 \%$ (12/53), 6\% (3/47), and $43 \%(3 / 7)$ of the isolates of E. coli, K. pneumoniae, and $K$. oxytoca, respectively, were included in this category. Among isolates presenting indeterminable results CAIE was evident in 5/12 E. coli, 2/3 K. pneumoniae, and $2 / 3$ Koxytoca. At the time this study was being performed, E-test strips containing a combination of CTX and CTX+CA were not available to us. Based on the results obtained by DDCT, it is expected that E-test strips with CTX and CTX-CA would contribute to demonstrate CAIE in a higher proportion of isolates. Using strips with CTX and $\mathrm{CTX}+\mathrm{CA}$ and $\mathrm{CAZ}$ and $\mathrm{CAZ}+\mathrm{CA}$, the occurrence of indeterminate results using the E-test was observed in $4 \%$ of isolates in a recent study [20].

What would be the impact of strains not confirmed by DDCT or E-test strip for clinical laboratories? Overall, due to the presence of a high number of false- positives in the screening procedure, the two steps strategy (screening/confirmatory tests) may result in increases in turnaround time and cost for the laboratory. Tests based on molecular detection of ESBL genes by PCR and isoelectric focusing [21,22] are more conclusive in defining ESBL production. These tests, however, are not available for most clinical laboratories. In an algorithm recently proposed [21], a disk diffusion test using cefoxitin is applied to those isolates of $K$. pneumoniae in which the CAIE is not verified. According to this algorithm, cefoxitin resistant isolates would likely have an alternative resistance mechanism, such as Amp-C-type enzyme and/or porin changes. On the other hand, susceptible isolates would suggest hyperproduction of ESBL. Isolates in which CAIE was not demonstrated by both DDCF and the E-test in our study were submitted to a cefoxitin susceptibility test and most organisms presented results with cefoxitin susceptibility, i.e., compatible with ESBL production (Tables 3 and 4). Our results show that the role of the cefoxitin susceptibility tests applied on strains that do not show the CAIE must be better defined.

Strains of E. coli and Klebsiella sp., may show resistance to $\beta$-lactam antibiotics by a diverse and continuously growing group of mechanisms. Clinical 
laboratories are constantly challenged by these strains and remain in need of a simple, straightforward method to detect ESBL production in E. coli and Klebsiella sp.

\section{Acknowledgments}

The authors wish to thank Dr. Beatriz Moreira, Instituto de Microbiologia, Universidade Federal do Rio de Janeiro, Rio de Janeiro, Brazil, for her cooperation. This study was supported in part by Conselho Nacional de Desenvolvimento Científico e Tecnológico (CNPq), Fundação Faculdade Federal de Ciências Médicas de Porto Alegre (FFFCMPA), and Complexo Hospitalar Irmandade Santa Casa de Misericórdia de Porto Alegre.

\section{References}

1. Gales A.C., Bolmstrom A., Sampaio J., et al. Antimicrobial susceptibility of Klebsiella pneumoniae producing extended-spectrum $\beta$-lactamases (ESBL) isolated in hospitals in Brazil. Braz J Infect Dis 1997;1:196-203.

2. Sader H.S., Sampaio J.L.M., Zoccoli C., Jones R.N.Results of the 1997 SENTRY antimicrobial surveillance program in three Brazilian medical centers. Braz J Infect Dis 1999;3:63-79.

3. Oplustil C.P., Nunes R., Mendes C. Multicenter evaluation of resistance patterns of Klebsiella pneumoniae, E. coli, Salmonella sp. and Shigella sp. isolated from clinical specimens in Brazil: Resistnet Surveillance Program. Braz J Infect Dis 2001;5:8-12.

4. Silva J., Gatica R., Aguilar C., et al. Outbreak of infection with extended-spectrum $\beta$-lactamase- producing Klebsiella pneumoniae in a Mexican Hospital. J Clin Microbiol 2001;39:3193-6.

5. Zemelman C., Bello H., Dominguez M., et al. Activity of cefepime, cefotaxime, ceftazidime, and aztreonam against extended-spectrum-producing isolates of Klebsiella pneumoniae and Escherichia coli from Chilean hospitals. Diagn Microbiol Infect Dis 2001;40:41-3.

6. Bell J.M., Turnidge J.D., Gales A.C., et al. Prevalence of extended spectrum $\beta$-lactamase (ESBL)- producing clinical isolates in the Asia-Pacific region and South Africa: regional results from SENTRY Antimicrobial Surveillance Program (1998-99). Diagn Microbiol Infect Dis 2002;42:193-8.
7. Bradford P.A. Extended-spectrum $\beta$-lactamases in the $21^{\text {st }}$ century: characterization, epidemiology, and detection of this important resistance threat. Clin Microbiol Rev 2001;4:933-51.

8. Bush K. New $\beta$-lactamases in Gram-negative bacteria: Diversity and impact on the selection of antimicrobial therapy. Clin Infect Dis 2001;32:1085-9.

9. Livermore D.M. $\beta$-lactamases in laboratory and clinical resistance. Clin Microbiol Rev 1995;8:557-84.

10. National Committee for Clinical Laboratory Standards. Performance standard for antimicrobial disk susceptibility testing; ninth informational supplement M100-S9. NCCLS, Wayne, PA, USA, 1999.

11. Bush K., Jacoby G.A., Medeiros A.A. A functional classification scheme for $\beta$-lactamases and its correlation with molecular structure. Antimicrob Agents Chemother 1995;39:1211-33.

12. Nordmann P. Trends in $\beta$-lactam resistance among Enterobacteriaceae. Clin Infect Dis 1998;27:S100-S6.

13. Ardanuy C., Liñares J., Dominguez M.A., et al. Outer membrane profiles of clonally related Klebsiella pneumoniae isolates from clinical samples and activities of cephalosporins and carbapenems. Antimicrob Agents Chemother 1998;42:1636-40.

14. Martínez-Martínez L., Hernández-Alléz S., Albertí S., et al. In vivo selection of porin-deficient mutants of Klebsiella pneumoniae with increased resistance to cefoxitin and expanded-spectrum cephalosporins. Antimicrob Agents Chemother 1996;40:342-8.

15. Martínez-Martínez L., Pascual A., Hernández-Alléz S., et al. Roles of $\beta$-lactamases and porins in activities of carbapenems and cephalosporins against Klebsiella pneumoniae. Antimicrob Agents Chemother, 1999;43:1669-73.

16. Chaibi E.B., Sirot D., Paul G., Labia R. Inhibitor-resistant TEM $\beta$-lactamases: phenotypic, genetic and biochemical characteristics. J Antimicrobs Chemother 1999;43:447-58.

17. Prinarakis E., Miriagou V., Tzelepi E., et al. Emergence of an inhibitor-resistant $\beta$-lactamase (SHV-10) derived from and SHV-5 variant. Antimicrob Agents Chemother 1997;41:838-40.

18. Jarlier V., Nicolas M., Fournier G., Philippon A. Extended broad-spectrum $\beta$-lactamases conferring transferable resistance to newer $\beta$-lactam agents in Enterobacteriaceae: hospital prevalence and susceptibility patterns. Rev Infect Dis 1998; 10 :867-78.

19. National Committee for Clinical Laboratory Standards. Performance standard for antimicrobial susceptibility testing. Eleventh informational supplement. Document M100-S10. NCCLS, Wayne, PA, USA, 2001. 
20. Leverstein-van Hall M.A., Fluit A.C., Paauw A., et al. Evaluation of the E-test ESBL and the BD Phoenix, Vitek 1, and Vitek 2 automated instruments for detection of Extended-Spectrum Beta-Lactamases in multiresistant Escherichia coli and Klebsiella sp.. J Clin Microbiol 2002; 40:3703-11.

21. Steward C.D., Rasheed J.K., Hubert S.K., et al. Characterization of clinical isolates of Klebsiella pneumoniae from 19 laboratories using the National Committee for Clinical Laboratory Standards ExtendedSpectrum $\beta$-lactamase detection methods. J Clin Microbiol 2001;39:2864-72.

22. Yu W.L., Pfaller M.A., Winokur P.L., Jones R.N. Cefepime MIC as a predictor of the Extended-Spectrum BetaLactamase type in Klebsiella penumoniae, Taiwan. Emer Infect Dis 2002;8:522-4. 